\title{
CARACTERISTICAS VOCAIS ACÚSTICAS DE HOMENS COM VOZ E LARINGE NORMAL
}

\section{Vocal acoustic characteristic in men with normal voice and laryngeal}

\author{
Bárbara Costa Beber ${ }^{(1)}$, Carla Aparecida Cielo (2)
}

\begin{abstract}
RESUMO
Tema: características vocais acústicas do filtro e das medidas da fonte vocal masculina. Objetivo: realizar uma revisão de literatura descrevendo e discutindo os aspectos que envolvem as características vocais acústicas de homens com laringe normal relacionadas às medidas da fonte e ao filtro vocal. Conclusão: as medidas de Jita, ShdB e NHR tendem a ser maiores nos homens, enquanto a fO, PHR/HNR e o ATRI tendem a ser menores. As espectrografias tendem a apresentar formantes graves, com média de alcance menor, com escurecimento variável e menos regular do que nas mulheres.
\end{abstract}

DESCRITORES: Voz; Saúde do Homem; Acústica da Fala

\section{INTRODUÇÃO}

A análise acústica é uma análise objetiva que faz uso de programas computadorizados e que, no Brasil, alcançou maior utilização na última década, quando os estudos na área se tornaram mais abrangentes. Essa análise permite avaliar a voz no início e no seguimento do tratamento fonoaudiológico, oferecendo várias ferramentas, dentre elas a análise das características acústicas da fonte glótica e a análise do filtro vocal, que é a espectrografia ${ }^{1}$. Para poder avaliar uma voz através de parâmetros numéricos, é preciso definir previamente a voz normal e dispor de valores normativos para tal comparação. No entanto, são escassos os estudos nesse sentido ${ }^{2}$.

(1) Fonoaudióloga da Prefeitura Municipal de Mostradas, RS e de Cidreira, RS; Especialista em Fonoaudiologia pela Universidade Federal de Santa Maria; Mestre em Distúrbios da Comunicação Humana pela Universidade Federal de Santa Maria.

(2) Fonoaudióloga; Professora Adjunta do Departamento de Fonoaudiologia da Universidade Federal de Santa Maria, UFSM, Santa Maria, RS; Aperfeiçoada em Voz pelo CEFAC - Pós-Graduação em Saúde e Educação; Especialista em Fonoaudiologia pela Universidade Federal de Santa Maria; Especialista em Linguagem pelo Conselho Federal de Fonoaudiologia; Mestre e Doutora em Linguística Aplicada pela Pontifícia Universidade Católica do Rio Grande do Sul.

Conflito de interesses: inexistente
A análise acústica fornece medidas que estão relacionadas ao padrão vibratório das pregas vocais, à forma do trato vocal, e às suas mudanças no tempo, e sua interpretação varia com a idade, o sexo, o tipo de fonação e o treino vocal. Assim, é notório que os resultados da mesma se diferenciem em cada população por fatores diversos ${ }^{3-6}$. No sexo masculino, os resultados da análise acústica seguirão determinados padrões que resultam de suas características anatômicas e fisiológicas. Nesse caso, alguns aspectos diferenciarão os resultados acústicos das vozes de homens, como a posição e o tamanho da laringe e do trato vocal, o comprimento e a largura das pregas vocais ${ }^{4,7,8}$.

Com base no que foi exposto, o objetivo deste estudo foi realizar uma revisão de literatura que apresentasse e discutisse os aspectos que envolvem as características vocais acústicas de homens com laringe normal, relacionadas tanto às medidas da fonte glótica como às características do filtro vocal.

\section{MÉTODO}

Para esta revisão de literatura, foi realizado um levantamento bibliográfico, sem data limite, utilizando livros, monografias, dissertações, teses, artigos de periódicos e Internet. Nesta última, foram pesquisados artigos pelo Lilacs, Bireme, PubMed, MedLine, Scielo, ISI e Google Schoolar. A consulta 
foi sistemática e em fontes clássicas e atuais da literatura científica nacional e internacional. Para a busca, foram utilizados os termos: voz masculina, voz no homem, análise acústica, espectrografia. Foram incluídos os estudos relevantes para os tópicos em questão e excluídos aqueles que apresentavam informações que não se enquadravam no estudo ou que apresentavam dados e informações de origem duvidosa. Os resultados da busca foram organizados, apresentados e discutidos em diferentes itens.

\section{REVISÃO DA LITERATURA}

\section{O aparelho fonador e a voz no homem}

A qualidade vocal de um sujeito é produto de dois processos que estão relacionados: a produção de um sinal vocal complexo pela laringe e a filtragem do mesmo pelo trato vocal. Alguns aspectos da voz são primários da laringe e outros são contribuições do filtro do trato vocal ${ }^{9-11}$.

Existem diferenças anatômicas e fisiológicas entre o organismo dos homens e das mulheres que resultam em características capazes de diferenciar os dois sexos. No caso das estruturas laríngeas, as pregas vocais dos homens são maiores e mais largas do que as das mulheres ${ }^{7}$. Existem diferenças quanto à posição vertical da laringe, que é mais baixa nos homens ${ }^{8}$. No entanto, tais variações são pouco conhecidas, mas sabe-se que elas permitem caracterizar as vozes quanto ao sexo.

As características estruturais dos elementos da face, como a forma, o tamanho, a densidade e a tensão têm influência sobre a produção vocal ${ }^{6,12}$. Estudos mostram que sujeitos com a face curta têm sua frequência de formantes deslocada para os agudos, enquanto naqueles com face longa os formantes tendem a se deslocar para os graves ${ }^{12}$.

Assim, a voz do homem é o resultado das características anatômicas e fisiológicas do seu aparelho fonador. O perfil vocal masculino pode ser explicado, basicamente, pelo fato das pregas vocais dos homens serem mais compridas e mais largas, suas laringes serem mais baixas, seu ângulo da cartilagem tireóide ser menor em relação às mulheres, e seu trato vocal ser maior e mais longo ${ }^{6-8}$.

\section{Características vocais acústicas de vozes masculinas: Medidas da fonte glótica}

Conhecer as características vocais acústicas de sujeitos normais é importante para estabelecer os padrões de normalidade que guiarão o profissional que estuda a voz. As vozes normais variam entre si, já que a voz é uma característica particular de cada sujeito, não existindo vozes perfeitamente iguais ${ }^{2,3,13}$.
Os laboratórios de voz que pesquisam as características vocais costumam trabalhar com inúmeras medidas acústicas, sendo que as principais e mais utilizadas são as medidas de frequência fundamental, de perturbação de frequência e de intensidade, de ruído, de tremor vocal, de quebras de voz, e de componentes subarmônicos. Cada uma dessas medidas pode variar de acordo com os aspectos individuais de cada sujeito, com o sexo e com a raça, e com as condições de fonação, além do tipo de extração da medida, segundo a grande maioria dos estudos na área ${ }^{4,14}$.

Nesta revisão de literatura, foram pesquisados programas de análise acústica que utilizassem a vogal /a/ para a extração das medidas acústicas e os programas encontrados foram o Multi Dimensional Voice Program (MDVP), Multi Dimensional Voice Program Advanced (MDVPA, que é a versão mais atualizada do MDVP), Computerized Speech Laboratory (CSL) e CSL 4300, que são todos programas produzidos pela empresa americana Kay Pentax e, portanto, apresentam parâmetros vocais da população americana. A empresa Kay Pentax vem desenvolvendo softwares para análise acústica da voz desde 1970. Também foram encontrados o Programa de Análise Acústica da Voz da Universidade de São Paulo (USP), desenvolvido pela Escola de Engenharia de São Paulo em 1998 e com parâmetros vocais da população brasileira ${ }^{3}$ e o WPCVox desenvolvido na Espanha pelo departamento de Circuitos e Sistemas da Universidade Politécnica de Madri, publicado em $2006{ }^{15}$.

Atualmente, o programa mais utilizado em pesquisas de análise acústica da voz é o MDVP, portanto seus padrões de normalidade serão utilizados para comparação no decorrer desta revisão, quando isto se fizer necessário.

As medidas de frequência fundamental são a própria frequência fundamental (fO) da voz, a média da f0 (Mf0), a f0 mais alta (fhi), a mais baixa (flo), e o desvio padrão da f0 (STD de f0). A fo fornece informações referentes à velocidade de vibração das pregas vocais e está relacionada com o comprimento, a tensão, a rigidez, e a massa das pregas vocais na sua interação com a pressão aérea subglótica, sendo mais grave nos homens 4,16,17. A f0 também é definida como o primeiro harmônico ${ }^{17}$ e é a medida mais utilizada pelos clínicos para caracterizar a voz humana, já que ela se distingue conforme a idade, o sexo, e a altura do indivíduo ${ }^{13,19}$

Estudos comprovam que a fo dos homens tende a aumentar com o avanço da idade ${ }^{20}$, havendo evidente aumento entre os homens na quinta e na oitava década de vida ${ }^{19}$. Ao contrário das afirmações anteriores, um estudo que pesquisou a fo em 
idosos de ambos os sexos encontrou declínio da fo no sexo masculino, embora esse declínio tenha sido menor do que o encontrado em mulheres ${ }^{21}$.

A Tabela 1 apresenta os principais valores encontrados na literatura para as medidas de frequência fundamental masculina. Alguns estudos fornecem, além das médias, faixas de normalidade. Determinado estudo obteve como faixa de normalidade os seguintes valores: f0 $83 \mathrm{a} 153 \mathrm{~Hz}$; fhi $85 \mathrm{a}$ $167 \mathrm{~Hz}$; flo 81 a $148 \mathrm{~Hz}$; e STDf0 0,73 a $4,46 \mathrm{~Hz}{ }^{2}$. Alguns autores são referência no Brasil quanto à faixa de normalidade da fo masculina que é de $80-150 \mathrm{~Hz}^{22}$.

Um estudo ${ }^{14}$ citado nas Tabelas 1 a 4 , traz medidas acústicas de vozes de sujeitos do sexo masculino com laringe normal, com idades de 9 a 79 anos. Os resultados deste estudo podem não ser adequados para caracterizar vozes masculinas, pois inclui indivíduos que não passaram pela muda vocal e, portanto, que não apresentam vozes propriamente masculinas. No entanto, o estudo foi mantido como revisão de literatura, pois foi a única publicação encontrada fornecendo medidas a partir do programa WpcVox.

Dentre os estudos de análise acústica, foi possível verificar que a extração da f0 não apresenta muitas discrepâncias entre os programas e que os resultados dos referidos estudos se localizam dentro da faixa clássica de normalidade proposta no Brasil ${ }^{22}$. O menor limite inferior encontrado para a f0 foi de $80 \mathrm{~Hz}{ }^{22}$ e o maior limite superior foi de $153 \mathrm{~Hz}^{2}$.

A fo da voz masculina é sua característica acústica mais marcante, pois é a medida que mais difere entre os sexos e a mais concordante entre os estudos de análise acústica ${ }^{2,3,14,16,18}$. A f0 masculina é mais grave, pois nas pregas vocais dos homens a massa que se coloca em vibração é maior do que a das mulheres, o que faz com que elas vibrem em uma velocidade mais lenta, gerando menos ciclos glóticos por segundo ${ }^{2}$. A grande maioria dos estudos afirma que a f0 dos homens tende a aumentar

Tabela 1 - Medidas de frequência fundamental da vogal /a/ para homens adultos

\begin{tabular}{|c|c|c|c|c|c|c|}
\hline Origem dos valores & $\begin{array}{l}\text { Programa } \\
\text { Utilizado }\end{array}$ & $\begin{array}{l}\text { Faixa } \\
\text { etária }\end{array}$ & $\begin{array}{l}\text { f0 }(\mathrm{Hz}) \\
\text { Média }\end{array}$ & $\begin{array}{l}\text { fhi }(\mathrm{Hz}) \\
\text { Média }\end{array}$ & $\begin{array}{l}\text { flo }(\mathrm{Hz}) \\
\text { Média }\end{array}$ & $\begin{array}{c}\text { STD f0 } \\
(\mathrm{Hz}) \text { Média }\end{array}$ \\
\hline $\begin{array}{c}\text { Dados de } \\
\text { normalidade do } \\
\text { MDVPA }\end{array}$ & MDVPA & - & 145,223 & 150,080 & 140,418 & 1,349 \\
\hline Fukuyama (2001) ${ }^{9}$ & MDVP & $20-58$ & 131,70 & - & - & - \\
\hline Araújo et al. $(2002)^{3}$ & $\begin{array}{c}\text { Programa de } \\
\text { Análise Acústica } \\
\text { da Voz (USP) }\end{array}$ & $20-40$ & 127,61 & - & - & - \\
\hline $\begin{array}{c}\text { González, Cervera e } \\
\text { Miralles (2002) }\end{array}$ & MDVP & $20-43$ & 120 & 125 & 115 & 1,35 \\
\hline $\begin{array}{c}\text { Wang e Huang } \\
(2004)^{30}\end{array}$ & CSL & $20-49$ & 121,27 & - & - & - \\
\hline $\begin{array}{c}\text { Guimarães e } \\
\text { Abberton (2005) }\end{array}$ & - & - & 118,4 & - & - & - \\
\hline Santos (2005) & $\begin{array}{c}\text { Programa de } \\
\text { Análise de Voz } 3.0 \\
\text { (USP) }\end{array}$ & $21-45$ & 134,50 & - & - & - \\
\hline $\begin{array}{l}\text { Smits, Ceuppen e } \\
\text { Bodt (2005) }\end{array}$ & $\mathrm{CSL}$ & $20-60$ & 115,25 & - & - & 1,42 \\
\hline $\begin{array}{l}\text { Felippe, Grillo e } \\
\text { Grechi (2006) }\end{array}$ & CSL 4300 & $20-45$ & 119,84 & - & - & - \\
\hline $\begin{array}{l}\text { Godino-Lhorente } \\
\text { et al. (2008) }\end{array}$ & WPCVox & $9-79$ & 119,70 & - & - & - \\
\hline $\begin{array}{l}\text { Godino-Lhorente } \\
\text { et al. (2008) }\end{array}$ & MDVP & $9-79$ & 120,56 & - & - & - \\
\hline $\begin{array}{l}\text { Yaser e Wingate } \\
(2008)^{31}\end{array}$ & MDVP & $18-24$ & - & - & - & - \\
\hline
\end{tabular}

Legenda - f0: Frequência fundamental, fhi: Frequência fundamental máxima, flo: Frequência fundamental mínima, STD f0: Desviopadrão da frequência fundamental

Fontes: autores citados dentro da tabela e programa MDVPA 
com o envelhecimento ${ }^{19,20}$ devido à atrofia dos músculos e tecidos da laringe, bem como pela perda de flexibilidade das pregas vocais ${ }^{16,19}$. O único estudo que não encontrou aumento da f0 nos homens ${ }^{21}$ baseou-se em um número reduzido de sujeitos e não levou em questão o uso vocal dos mesmos, fator que poderia influenciar a f0 desses sujeitos.

Os dados obtidos da avaliação de quanto um período da onda sonora se diferencia do outro que o sucede, com relação à frequência e à intensidade, denominam-se medidas de perturbação da frequência e da intensidade. Esses dados se relacionam com a instabilidade do sinal, ciclo-a-ciclo ou em curto termo, e com o controle sobre o sistema fonatório. A perturbação da frequência ciclo-aciclo denomina-se Jitter e a perturbação da intensidade ou da amplitude ciclo-a-ciclo denomina-se Shimmer, sendo que existem diferentes formas de extração dessas medidas $4,14,16,17,23$.

As medidas de perturbação da freqüência, pelo MDVPA, são o Jitter absoluto (Jita); Jitter percentual (Jitt); média relativa da perturbação (RAP); quociente de perturbação do pitch (PPQ); quociente de perturbação do pitch suavizado (SPPQ); coeficiente da variação da frequência fundamental (vf0). As medidas de perturbação da intensidade, pelo MDVPA, são o Shimmer em dB (ShdB); Shimmer percentual (Shim); quociente de perturbação da amplitude (APQ); quociente de perturbação da amplitude suavizado (sAPQ); coeficiente de variação da amplitude (vAm) ${ }^{4,16}$. As medidas de perturbação da amplitude são consideradas por alguns autores como as mais significativas para determinar a severidade das disfonias ${ }^{23}$.

O Shimmer é frequentemente associado ao ruído na produção vocal, portanto tende a ser mais alto nos casos de soprosidade. Já o Jitter tende a ser mais alto nos casos de alteração nas pregas vocais ${ }^{24}$.

As medidas de Jitter e Shimmer podem variar conforme a vogal analisada, a idade e o sexo do sujeito e, quanto ao Jitter, um trabalho coloca que ele pode variar também conforme a língua falada, mas não justifica a afirmação ${ }^{16}$. Este fato deveria ser levado em consideração quando são utilizados programas que já forneçam um padrão de normalidade baseado em uma língua diferente da língua da população que está sendo estudada.

O Jitter e o Shimmer absolutos, na maioria das pesquisas, mostram-se maiores para o sexo masculino, embora nem sempre haja diferença estatisticamente significante ${ }^{2,14,17,22,23}$, ocorrendo o mesmo com os padrões de normalidade utilizados pelo programa MDVP. Um maior valor de Jitter indica menos controle sobre a voz e alguns autores referem que isto pode estar relacionado ao fato dos homens fazerem menos uso vocal do que as mulheres, evidenciando, então, menos treino vocal e conseqüente maior instabilidade ${ }^{22}$, além das características anatômicas da laringe masculina, com maior extensão e volume das pregas vocais. O Jitter aparece também correlacionado à presença de ruído na voz ou à rouquidão ${ }^{16}$. Portanto, quando os achados de valores de Jitter aparecem maiores nos homens do que nas mulheres ${ }^{2,14,17,22,23}$, eles estão de acordo com os achados de valores de medidas de ruído, que também tendem a ser maiores no sexo masculino ${ }^{3,2,17}$, como observado na presente revisão de literatura.

As medidas de Jittere Shimmer tendem a mudar com o envelhecimento. Nos homens, é esperado que ocorra um aumento tanto do Jitter como do Shimmer ${ }^{19,20}$. O achado se justifica pelas alterações anatômicas e fisiológicas que ocorrem na laringe com a idade, como a atrofia dos músculos e a perda de flexibilidade das pregas vocais, que aumentam a instabilidade vocal ${ }^{16}$.

Apenas um estudo discorda da maioria da literatura, quando afirma que o Shimmer diminui com a idade, provavelmente devido ao fato dos sujeitos participantes da pesquisa serem física e socialmente ativos ${ }^{19}$.

Quando o Shimmer aparece aumentado com o passar dos anos, decorre das alterações anatômicas e fisiológicas do envelhecimento que, assim como no caso do Jitter, aumentam a instabilidade do sinal ${ }^{20}$. Porém, ainda há muito a ser estudado quando o assunto é envelhecimento vocal e laríngeo.

É importante normalizar os valores de Jitter e Shimmer para vogais sustentadas, pois os mesmos aparecem significativamente diferentes entre sujeitos normais e sujeitos com patologias laríngeas ${ }^{23,25}$. Os valores médios de normalidade para homens encontrados pelos autores pesquisados encontramse nas Tabelas 2 e 3 . Além das médias, o programa MDVPA fornece um limiar superior (threshold) que, para as medidas de perturbação de freqüência e de intensidade masculinas, são: Jita 83,2 us, Jitt $1,040 \%$, RAP 0,680\%, PPQ 0,840\%, sPPQ 1,020\%, e vf0 1,100\%; ShdB 0,350dB, Shim 3,810\%, APQ $3,070 \%$, sAPQ $4,230 \%$, e vAm $8,200 \%$. Um estudo fornece faixas de normalidade para essas medidas que são as seguintes: Jita 20,57 a 167,39us; Jitt 0,25 a $2,14 \%$; RAP 0,14 a $1,31 \%$; PPQ 0,15 a $1,21 \%$; sPPQ 0,38 a $1,52 \%$; vf0 0,60 a $3,26 \%$; ShdB 0,11 a 0,74dB; Shim 1,33 a 8,33\%; APQ 1,34 a 7,06\%; sAPQ 2,69 a 9,31\%; vAm 4,75 a31,43\% ${ }^{2}$. Ainda, outra pesquisa encontrou os seguintes valores para as medidas de perturbação da intensidade em homens: ShdB 0,227 a 0,354dB; Shim 2,532 a 3,011\%; APQ 2,084 a 3,101\%; sAPQ 2,495 a 
Tabela 2 - Medidas de perturbação de frequência da vogal /a/ para homens adultos

\begin{tabular}{|c|c|c|c|c|c|c|c|c|}
\hline $\begin{array}{l}\text { Origem } \\
\text { dos } \\
\text { Valores }\end{array}$ & $\begin{array}{c}\text { Programa } \\
\text { Utilizado }\end{array}$ & $\begin{array}{l}\text { Faixa } \\
\text { Etária }\end{array}$ & $\begin{array}{l}\text { Jita } \\
\text { (us) } \\
\text { Média }\end{array}$ & $\begin{array}{c}\text { Jitt } \\
\text { (\%) } \\
\text { Média }\end{array}$ & $\begin{array}{l}\text { RAP } \\
(\%) \\
\text { Média }\end{array}$ & $\begin{array}{l}\text { PPQ (\%) } \\
\text { Média }\end{array}$ & $\begin{array}{c}\text { sPPQ } \\
(\%) \\
\text { Média }\end{array}$ & $\begin{array}{l}\text { vf0 (\%) } \\
\text { Média }\end{array}$ \\
\hline $\begin{array}{c}\text { Dados de } \\
\text { normalidade } \\
\text { do MDVPA }\end{array}$ & MDVPA & - & 41,663 & 0,589 & 0,345 & 0,338 & 0,561 & 0,939 \\
\hline $\begin{array}{c}\text { Fukuyama } \\
(2001)^{9}\end{array}$ & MDVP & $20-58$ & - & - & - & 0,35 & - & - \\
\hline $\begin{array}{l}\text { Araújo et al. } \\
\qquad(2002)^{3}\end{array}$ & $\begin{array}{c}\text { Programa } \\
\text { de Análise } \\
\text { Acústica } \\
\text { da Voz } \\
\text { (USP) }\end{array}$ & $20-40$ & - & 0,37 & - & 0,30 & - & - \\
\hline $\begin{array}{c}\text { González, } \\
\text { Cervera e } \\
\text { Miralles } \\
(2002)^{2}\end{array}$ & MDVP & $20-43$ & 57,53 & 0,68 & 0,39 & 0,40 & 0,68 & 1,12 \\
\hline $\begin{array}{l}\text { Nicastri et } \\
\text { al. }(2004)^{22}\end{array}$ & MDVP & $19-59$ & - & - & - & - & 2,61 & 3,43 \\
\hline $\begin{array}{l}\text { Wang e } \\
\text { Huang } \\
(2004)^{30}\end{array}$ & CSL & $20-49$ & - & 0,56 & - & - & - & - \\
\hline $\begin{array}{l}\text { Santos } \\
(2005)^{19}\end{array}$ & $\begin{array}{c}\text { Programa } \\
\text { de Análise } \\
\text { de Voz } 3.0 \\
\text { (USP) }\end{array}$ & $21-45$ & - & 0,82 & - & - & - & - \\
\hline $\begin{array}{c}\text { Smits, } \\
\text { Ceuppens e } \\
\text { Bodt } \\
(2005)^{18}\end{array}$ & CSL & $20-60$ & 64,245 & 0,73 & - & - & - & - \\
\hline $\begin{array}{l}\text { Felippe, } \\
\text { Grillo e } \\
\text { Grechi } \\
(2006)^{17}\end{array}$ & CSL 4300 & $20-45$ & - & 0,49 & - & - & - & - \\
\hline $\begin{array}{l}\text { Godino- } \\
\text { Lhorente et } \\
\text { al. }(2008)^{14}\end{array}$ & WPCVox & $9-79$ & 72,25 & 0,84 & 0,49 & 0,49 & 0,80 & - \\
\hline $\begin{array}{l}\text { Godino- } \\
\text { Lhorente et } \\
\text { al. }(2008)^{14}\end{array}$ & MDVP & $9-79$ & 77,08 & 0,96 & 0,57 & 0,56 & 0,86 & - \\
\hline
\end{tabular}

Legenda: Jita: Jitter absoluto, Jitt: Jitter percentual, RAP: Média relativa da perturbação, PPQ: Quociente de perturbação do pitch, SPPQ: Quociente de perturbação do pitch suavizado, vf0: Coeficiente da variação da freqüência fundamental.

Fontes: autores citados dentro da tabela e programa MDVPA.

$5,002 \%$ e vAm 5,892 a $7,262 \%{ }^{23}$. É possível observar que os limiares superiores encontrados pelo primeiro estudo² são visivelmente maiores do que os limiares fornecidos pelo MDVPA e uma possibilidade de justificativa para essa diferença seria o fato de os sujeitos serem falantes do espanhol e não do inglês, como os que participaram da padronização do MDVPA. Já no segundo estudo ${ }^{23}$, os valores dos limiares superiores encontrados ficaram mais próximos do MDVPA, no entanto os sujeitos desta pesquisa também não eram falantes do inglês, e sim do italiano.

As medidas de ruído medem a presença de ruído em variadas faixas do espectro vocal ou no espectro como um todo, e relacionam-se a vozes alteradas. Também tornam possível obter informações sobre a relação entre o componente harmônico e o componente de ruído da onda sonora. 
Tabela 3 - Medidas de perturbação da intensidade da vogal /a/ para homens adultos

\begin{tabular}{|c|c|c|c|c|c|c|c|}
\hline $\begin{array}{l}\text { Origem dos } \\
\text { Valores }\end{array}$ & $\begin{array}{l}\text { Programa } \\
\text { Utilizado }\end{array}$ & $\begin{array}{l}\text { Faixa } \\
\text { Etária }\end{array}$ & $\begin{array}{l}\text { ShdB(dB) } \\
\text { Média }\end{array}$ & $\begin{array}{c}\text { Shim(\%) } \\
\text { Média }\end{array}$ & $\begin{array}{l}\text { APQ(\%) } \\
\text { Média }\end{array}$ & $\begin{array}{l}\text { SAPQ (\%) } \\
\text { Média }\end{array}$ & $\begin{array}{c}\text { vAm(\%) } \\
\text { Média }\end{array}$ \\
\hline $\begin{array}{l}\text { Dados de } \\
\text { normalidade do } \\
\text { MDVPA }\end{array}$ & MDVPA & - & 0,219 & 2,523 & 1,986 & 3,055 & 7,712 \\
\hline $\begin{array}{c}\text { Fukuyama } \\
(2001)^{9}\end{array}$ & MDVP & $20-58$ & - & - & 2,30 & - & - \\
\hline $\begin{array}{c}\text { González, } \\
\text { Cervera e } \\
\text { Miralles }(2002)^{2}\end{array}$ & MDVP & $20-43$ & 0,33 & 3,82 & 3,06 & 4,81 & 12,38 \\
\hline $\begin{array}{l}\text { Nicastri et al. } \\
\quad(2004)^{23}\end{array}$ & MDVP & $19-59$ & 0,269 & 2,892 & 2,611 & 3,433 & 6,385 \\
\hline $\begin{array}{l}\text { Wang e Huang } \\
\qquad(2004)^{30}\end{array}$ & CSL & $20-49$ & 0,22 & - & - & - & - \\
\hline Santos $(2005)^{19}$ & $\begin{array}{l}\text { Programa de } \\
\text { Análise de } \\
\text { Voz } 3.0 \text { (USP) }\end{array}$ & $21-45$ & - & 6,06 & - & - & - \\
\hline $\begin{array}{c}\text { Smits, } \\
\text { Ceuppens e } \\
\text { Bodt }(2005)^{18}\end{array}$ & CSL & $20-60$ & - & 4,777 & - & - & - \\
\hline $\begin{array}{l}\text { Felippe, Grillo e } \\
\text { Grechi }(2006)^{17}\end{array}$ & CSL 4300 & $20-45$ & 0,22 & - & - & - & - \\
\hline $\begin{array}{l}\text { Godino- } \\
\text { Lhorente et al. } \\
(2008)^{14}\end{array}$ & WPCVox & $9-79$ & 0,31 & 3,53 & 2,75 & 5,12 & - \\
\hline $\begin{array}{l}\text { Godino- } \\
\text { Lhorente et al. } \\
(2008)^{14}\end{array}$ & MDVP & $9-79$ & 0,39 & 3,86 & 2,98 & 5,34 & - \\
\hline
\end{tabular}

Legenda - ShdB: Shimmer em decibels, Shim: Shimmer percentual, APQ: Quociente de perturbação da amplitude, sAPQ: Quociente de perturbação do pitch suavizado, vAm: Coeficiente de variação da amplitude.

Fontes: autores citados dentro da tabela e programa MDVPA.

As principais medidas relacionadas ao ruído são: proporção ruído-harmônico (NHR); índice de turbulência da voz (VTI); índice de fonação suave (SPI); proporção harmônico-ruído (PHR ou HNR) ${ }^{4,16}$.

As medidas NHR e HNR/PHR são medidas inversamente proporcionais e variam conforme o programa de análise acústica utilizado. Quanto menor for a NHR e maior for a HNR/PHR, melhor será a qualidade vocal, sendo que tais medidas refletem a avaliação geral do ruído no sinal analisado, não sendo específicas para ciclos determinados; incluem contribuições tanto das perturbações de amplitude quanto de frequência e são medidas para a determinação da percepção geral de ruído e de rouquidão no sinal vocal ${ }^{4,14,16}$. Estudos sobre a PHR encontraram diferença significativa entre os sexos, sendo a PHR menor para o sexo masculino ${ }^{3,17}$. Também houve diferença estatisticamente significante com o NHR maior nos homens noutro trabalho ${ }^{2}$. Isso ocorre, provavelmente pelo fato dos homens utilizarem mais a voz fluida e o registro basal, que são caracterizados por menor força de coaptação glótica, favorecendo diminuição dos harmônicos e mais ruído glótico ${ }^{16}$, além das medidas de jitter serem naturalmente maiores nos homens, o que pode também influenciar o nível de ruído nessas vozes, como foi comentado anteriormente, quando se falou da relação entre Jitter, ruído e rouquidão.

Algumas médias de normalidade encontradas na literatura estão evidenciadas na Tabela 4. Os limites superiores do MDVPA para as medidas de ruído são: NHR 0,190; VTI 0,061 e SPI 14,120. As seguintes faixas de normalidade são propostas por um estudo: NHR 0,10 a 0,23; VTI 0,02 a 0,10; SPI 2,87 a $17,51^{2}$. Os limites superiores deste estudo apareceram ligeiramente acima dos do MDVPA.

As medidas de quebra de voz do MDVP podem verificar o grau de quebra pelo DVB (grau de quebra da voz) e o número de quebras vocais (NVB) na amostra analisada. Essas medidas estão relacionadas à irregularidade da emissão, a áreas da amostra não-harmônicas ou à interrupção da produção vocal ${ }^{4}$. 
Beber BC, Cielo CA

Tabela 4 - Medidas de Ruído da vogal /a/ para homens adultos

\begin{tabular}{|c|c|c|c|c|c|c|}
\hline $\begin{array}{c}\text { Origem dos } \\
\text { Valores }\end{array}$ & $\begin{array}{c}\text { Programa } \\
\text { Utilizado }\end{array}$ & $\begin{array}{l}\text { Faixa } \\
\text { Etária }\end{array}$ & $\begin{array}{c}\text { HNR ou } \\
\text { PHR Média }\end{array}$ & $\begin{array}{c}\text { NHR } \\
\text { Média }\end{array}$ & $\begin{array}{c}\text { VTI } \\
\text { Média }\end{array}$ & SPI Média \\
\hline $\begin{array}{c}\text { Dados de } \\
\text { normalidade do } \\
\text { MDVPA }\end{array}$ & MDVPA & & & 0,122 & & 6,770 \\
\hline $\begin{array}{c}\text { Fukuyama } \\
(2001)^{9}\end{array}$ & MDVP & $20-58$ & & 0,15 & 0,05 & 10,72 \\
\hline $\begin{array}{l}\text { Araújo et al. } \\
(2002)^{3}\end{array}$ & $\begin{array}{c}\text { Programa de } \\
\text { Análise } \\
\text { Acústica da } \\
\text { Voz (USP) }\end{array}$ & $20-40$ & 1,06 & & & \\
\hline $\begin{array}{c}\text { González, } \\
\text { Cervera e } \\
\text { Miralles }(2002)^{2}\end{array}$ & MDVP & $20-43$ & & 0,14 & 0,05 & 8,78 \\
\hline $\begin{array}{l}\text { Wang e Huang } \\
\qquad(2004)^{30}\end{array}$ & CSL & $20-49$ & 10,13 & & & \\
\hline $\begin{array}{c}\text { Smits, } \\
\text { Ceuppens e } \\
\text { Bodt }(2005)^{18}\end{array}$ & CSL & $20-60$ & 16,315 & & & \\
\hline $\begin{array}{l}\text { Felippe, Grillo e } \\
\text { Grechi }(2006)^{17}\end{array}$ & CSL 4300 & $20-45$ & 9,56 & & & \\
\hline $\begin{array}{l}\text { Godino- } \\
\text { Lhorente et al. } \\
(2008)^{14}\end{array}$ & MDVP & $9-79$ & & 0,13 & 0,04 & \\
\hline $\begin{array}{l}\text { Godino- } \\
\text { Lhorente et al. } \\
(2008)^{14}\end{array}$ & WPCVox & $9-79$ & & 1,14 & 0,70 & \\
\hline
\end{tabular}

Legenda - HNR ou PHR: Proporção harmônico-ruído, NHR: Proporção ruído-harmônico, VTI: Índice de turbulência da voz, SPI: Índice de fonação suave.

Fontes: autores citados dentro da tabela e programa MDVPA.

Também existem as medidas de segmentos surdos, que são o número de segmentos não sonorizados (NUV) e o grau de silêncio ou período sem voz (DUV), que caracterizam a interrupção da periodicidade da onda sonora, refletindo-se como irregularidades ou ruído à emissão ${ }^{2,4,26}$.

As medidas de componentes subarmônicos permitem medir a presença de componentes subarmônicos de baixa intensidade situados entre os harmônicos por meio das medidas: Número de segmentos sub-harmônicos (NSH) e Grau dos componentes sub-harmônicos (DSH). Tais medidas sugerem irregularidade devido à vibração de outras estruturas (diplofonia) ou presença de vocal fry (flutuação), quando maiores; e ruído generalizado, quando menores (perturbação) ${ }^{2,4,26}$.

A Tabela 5 apresenta os valores encontrados na literatura para as medidas de quebra de voz, de segmentos surdos e de componentes subarmônicos. Para essas medidas, o MDVPA fornece como limiar superior: DVB, DSH e DUV 1,000\%; NVB, NSH e NUV 0,900.
Um trabalho ${ }^{2}$ encontrou como faixas de normalidade: DUV 0-24,49 \%, NUV 0-24\%, DSH 0-2\% e $\mathrm{NSH} 0-2 \%$. Os mesmos autores compararam as médias masculinas com as médias femininas e observaram diferença significativa entre os sexos em relação ao $\mathrm{DSH}$ e ao $\mathrm{NSH}$, sendo menores nos homens. Já o DUV e o NUV mostraram-se maiores no sexo masculino, porém sem diferença significativa. Neste estudo, as médias e faixas de normalidade obtidas para tais medidas estiveram todas dentro da normalidade proposta pelo MDVPA.

Existem, também, as medidas de tremor vocal que estão ligadas à variação de longo termo da frequência e da amplitude do sinal vocal: frequência de tremor da frequência ( $F f t r)$; frequência de tremor da amplitude (Fatr); índice de intensidade da frequência do tremor (FTRI), que evidencia a relação entre a f0 do tremor e a f0 da amostra; índice de intensidade da amplitude do tremor (ATRI), que mostra a relação entre a amplitude do tremor e a amplitude da amostra ${ }^{4,26}$. Um estudo encontrou valores de ATRI significativamente menores nos homens ${ }^{2}$. 
Tabela 5 - Medidas de quebras de voz, de segmentos surdos e de componentes subarmônicos da vogal /a/ para homens adultos

\begin{tabular}{|c|c|c|c|c|c|c|c|c|}
\hline $\begin{array}{l}\text { Origem dos } \\
\text { Valores }\end{array}$ & $\begin{array}{l}\text { Programa } \\
\text { Utilizado }\end{array}$ & $\begin{array}{l}\text { Faixa } \\
\text { Etária }\end{array}$ & $\begin{array}{l}\text { DVB } \\
(\%) \\
\text { Média }\end{array}$ & $\begin{array}{l}\text { NVB } \\
\text { Média }\end{array}$ & $\begin{array}{l}\text { DUV } \\
(\%) \\
\text { Média }\end{array}$ & $\begin{array}{l}\text { NUV } \\
\text { Média }\end{array}$ & $\begin{array}{l}\text { DSH } \\
(\%) \\
\text { Média }\end{array}$ & $\begin{array}{l}\text { NSH } \\
\text { Média }\end{array}$ \\
\hline $\begin{array}{l}\text { Dados de } \\
\text { normalidade do } \\
\text { MDVPA }\end{array}$ & MDVPA & & 0,200 & 0,200 & 0,200 & 0,200 & 0,200 & 0,200 \\
\hline $\begin{array}{c}\text { González, } \\
\text { Cervera e } \\
\text { Miralles }(2002)^{2}\end{array}$ & MDVP & $20-43$ & - & - & 0,55 & 0,53 & 0,07 & 0,06 \\
\hline
\end{tabular}

Tabela 6 - Medidas de tremor vocal da vogal /a/ para homens adultos

\begin{tabular}{|c|c|c|c|c|c|c|}
\hline Origem dos Valores & $\begin{array}{c}\text { Programa } \\
\text { Utilizado }\end{array}$ & $\begin{array}{l}\text { Faixa } \\
\text { Etária }\end{array}$ & $\begin{array}{l}\text { Fftr }(\mathbf{H z}) \\
\text { Média }\end{array}$ & $\begin{array}{l}\text { Fatr }(\mathrm{Hz}) \\
\text { Média }\end{array}$ & $\begin{array}{l}\text { FTRI (\%) } \\
\text { Média }\end{array}$ & $\begin{array}{l}\text { ATRI (\%) } \\
\text { Média }\end{array}$ \\
\hline $\begin{array}{l}\text { Dados de } \\
\text { normalidade do } \\
\text { MDVPA }\end{array}$ & MDVPA & & 3,655 & 2,728 & 0,311 & 2,133 \\
\hline 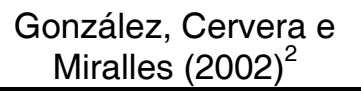 & MDVP & $20-43$ & 2,071 & 2,072 & 0,39 & 3,98 \\
\hline
\end{tabular}

Legenda - Fftr: Frequência de frequência do tremor, Fatr: Frequência da amplitude do tremor, FTRI: Índice da frequência do tremor, ATRI: Índice da amplitude do tremor.

Fontes: autores citados dentro da tabela e programa MDVPA.

A Tabela 6 mostra as médias de normalidade dos estudos revisados para as medidas de tremor vocal encontradas na literatura. O MDVPA indica como limiar superior para essas medidas nos homens: FTRI 0,950\%; ATRI 4,370\%. É importante salientar que, assim como para as medidas de frequência, para o Fftr e para o Fatr, o programa não fornece o limiar superior. Em pesquisa ${ }^{2}$, encontrou-se, como faixa de normalidade: Fftr 1,01 a 12,50Hz; Fatr 1,05 a $7,54 \mathrm{~Hz}$; FTRI 0,13 a 0,86\%; ATRI 0,92 a $13,85 \%$.

As medidas de tremor vocal, em especial o ATRI, mostraram-se menores no sexo masculino apenas em um estudo ${ }^{2}$, não sendo justificada pelos autores. No entanto, considerando-se que tais medidas podem aparecer mesmo em vozes classificadas como normais, em função de que o sinal glótico não é totalmente periódico, poder-se-ia inferir que os homens apresentam maior estabilidade fonatória de longo termo, sustentando melhor a emissão.

Para poder avaliar a voz disfônica por meio de parâmetros numéricos, é importante definir previamente a voz normal e dispor de valores normalizados para comparação, no entanto ainda são poucos os estudos sobre essa questão ${ }^{1}$. Por outro lado, alguns valores podem ser dependentes dos algoritmos usados pelo software específico que os calcula e alguns algoritmos utilizados podem não trabalhar bem para diferentes interlocutores e seu rendimento pode sofrer interferência do nível de ruído ${ }^{26}$.

Este trabalho procurou apresentar as faixas de normalidade e as médias das medidas acústicas encontradas em diferentes estudos a partir da análise acústica da vogal /a/ em vozes masculinas previamente definidas como normais e em sujeitos sem alterações laríngeas. Esses valores poderão ser utilizados como referência, conforme cada pesquisa exposta, para os profissionais da voz, assim como para melhor compreender, a partir das discussões anteriores, as possíveis justificativas para achados inesperados no estudo das vozes masculinas.

\section{Características vocais acústicas de vozes masculinas: Características espectrográficas do filtro}

A avaliação acústica baseia-se no Teorema de Fourier o qual afirma que qualquer forma de onda periódica pode ser analisada ou decomposta em uma série de ondas sinusóides. A onda vocal é uma onda quase periódica formada por várias ondas 
sinusoides de diferentes frequências. As frequências de suas ondas componentes são chamadas de harmônicos (h) e sempre serão múltiplos inteiros da f0 da onda, que será sempre a mais grave do espectro ${ }^{16}$.

Outras partes do trato vocal como a faringe, a cavidade oral e a nasal, também participam da determinação da qualidade vocal, atuando como filtro vocal. Essas estruturas atuam como ressonadores que amortecem ou amplificam certas frequências, determinando os formantes (F) que são grupos ou zonas de freqüências ou de harmônicos que recebem maior reforço de intensidade do trato vocal ${ }^{10-11,16}$. Diversos estudos relatam a ação modificadora do filtro vocal sobre o sinal vocal produzido pelas pregas vocais ${ }^{9-11,16}$. A espectrografia pode ser descrita como um gráfico tridimensional que apresenta em sua ordenada a frequência, em sua abscissa o tempo, e seu grau de escurecimento do traçado relaciona-se com a intensidade. Ela reflete tanto dados relativos à fonte glótica como dados da postura do trato vocal, podendo caracterizar vogais e consoantes. A avaliação do traçado espectrográfico informa sobre a natureza da fonte sonora e a contribuição do sistema de ressonância, fornecendo dados em dois aspectos: a distribuição dos harmônicos no espectro e análise dos formantes do som 1,4,16,27. O estudo da distribuição dos harmônicos se refere à ressonância e à projeção vocal. Quanto maior o número de harmônicos, mais rica é a qualidade vocal e, quanto mais individualizados e lineares, maior o componente harmônico da emissão e a estabilidade da sustentação, e menor a quantidade de energia aperiódica ou ruído ${ }^{1}$.

Quanto à análise dos formantes, quando há interrupção, irregularidade, escurecimento variável e largura de banda aumentada, estarão sendo compatíveis com uma voz ruidosa, com instabilidade da fonte glótica e com pouco aproveitamento da ressonância ${ }^{16}$.

Existem filtros de banda que transmitem frequências dentro de uma faixa estabelecida, atenuando determinadas frequências ${ }^{4}$. O filtro de banda estreita atinge a faixa de análise entre 15 e $60 \mathrm{~Hz}$; sua análise é lenta, porém mostra com precisão as frequências do componente do sinal (harmônicos e padrão de entonação); sua representação dispõe de estrias horizontais e, quanto mais adequada a voz, mais definidos estarão os harmônicos. O filtro de banda estreita, mostra a presença de subharmônicos, ruído entre os harmônicos, tremor ou instabilidade ${ }^{4}$. Alguns autores afirmam que o filtro de banda larga analisa as frequências entre $50 \mathrm{e}$ $600 \mathrm{~Hz}{ }^{4}$, outros afirmam que analisa entre 150 e $600 \mathrm{~Hz}{ }^{16}$. A resposta do filtro de banda larga é rápida, mas a resolução das frequências não é boa.
Sua vantagem é mostrar com clareza os pontos de mudança temporal (formantes), analisar a periodicidade da voz e a qualidade vocal ${ }^{4}$, permitindo também obter informações quanto às características articulatórias ${ }^{16}$.

A idade e o sexo podem ser determinantes do comprimento e do formato do trato vocal. Como os homens têm um trato vocal mais longo, apresentam frequências de formantes mais graves, portanto a energia aparece mais concentrada na parte inferior do espectro ${ }^{5}$. Frequências formantes de vogais tendem a apresentar decréscimo com o avanço da idade ${ }^{5,20}$.

Um estudo analisou a relação entre frequências formantes e medidas do corpo de sujeitos adultos. Os resultados mostraram que há pouca relação entre as medidas do corpo de adultos do mesmo sexo e seus formantes, sendo que essa relação é menor ainda no sexo masculino. No entanto, o autor comenta que outros estudos encontraram relação quando essas variáveis foram comparadas entre os sexos e, também, que há um decréscimo dos formantes com o crescimento da criança até a idade adulta ${ }^{5}$.

As características espectrográficas também podem variar conforme a população estudada. Um estudo analisou a qualidade vocal e os formantes das vogais de sujeitos adultos de uma cidade do nordeste do Brasil. As medidas do primeiro (F1), segundo (F2) e terceiro formantes (F3) encontradas mostraram diferenças estatisticamente significantes, quando comparadas com estudos de outras regiões do país tomados como referência, sendo que os valores de F1, F2 e F3 foram significativamente menores para os homens e significativamente maiores para as mulheres ${ }^{28}$.

Outro estudo encontrou diferenças nas frequências $F$ de diferentes raças, as quais apresentam características morfológicas diferentes. As diferenças encontradas estavam diretamente relacionadas com medidas como o comprimento e volume da cavidade oral, da faringe e da laringe ${ }^{6}$.

Outra pesquisa analisou vozes normais, roucas e ásperas de sujeitos do sexo masculino e do sexo feminino por meio da espectrografia. Quando analisados os dados do sexo masculino, pôde-se perceber um discreto rebaixamento da frequência naqueles com vozes roucas e um aumento acentuado naqueles com vozes ásperas. Os casos com vozes masculinas normais preencheram todo ou quase todo o intervalo do espectrograma com uma média de alcance de $4,24 \mathrm{KHz}$, mostrando riqueza de harmônicos e um alcance discretamente menor que o das mulheres. Quanto à configuração dos harmônicos, nas vozes roucas de ambos os sexos, eles apareceram mal definidos com instabilidade e 
baixa amplitude, enquanto que, nas vozes ásperas, a definição foi moderada e o escurecimento entre eles foi mais homogêneo. Os casos normais apresentaram redução de ruído entre as linhas dos harmônicos mais agudos, mostrando maior periodicidade da onda. Ainda, foi possível perceber que em todos os casos de vozes alteradas houve ausência de harmônicos acima de $3 \mathrm{KHz}{ }^{1}$.

Espera-se que, na espectrografia de banda larga de vozes masculinas, os formantes sejam mais graves, estando deslocados mais para a parte inferior do espectro ${ }^{5,29}$, pois, como afirmaram alguns autores ${ }^{12}$, em sujeitos de face longa os formantes tendem a se deslocar para os graves, e os homens tendem a apresentar faces mais longas do que as mulheres e, consequentemente, trato vocal mais longo.

Quanto à espectrografia de banda estreita, podese esperar harmônicos mais graves, pois é sabido que os harmônicos são múltiplos inteiros da f0 que também é mais grave nos homens ${ }^{16}$, pela própria característica anatômica de suas pregas vocais e da laringe ${ }^{7}$. Estas suposições vão ao encontro do estudo ${ }^{1}$ que encontrou uma média de alcance menor nos espectros masculinos.

Como discutido anteriormente, os valores de Jittere Shimmer costumam ser maiores para as vozes masculinas, o que significa maior instabilidade de frequência e de intensidade de curto termo do sinal vocal. Desta forma, pode-se esperar certa instabilidade também em seus espectros vocálicos, o que leva a pensar em uma espectrografia com harmônicos menos lineares e escurecimento mais variável e menos regular ${ }^{4,16}$.

Os estudos que encontraram NHR maior e PHR menor nos homens indicam que as vozes masculinas são mais ruidosas, o que também poderá ser percebido na análise espectrográfica, que pode ser caracterizada por perda de distinção dos harmônicos, chuviscos no traçado e perda de nitidez do gráfico ${ }^{16}$.

Ainda, em estudo ${ }^{28}$ sobre características espectrográficas confirma resultados de outras pesquisas de que as vozes variam conforme a população e a raça ${ }^{3,4,6}$. Este achado também vai ao encontro de estudos das medidas acústicas da fonte vocal que foram realizados em diferentes populações ${ }^{2,13,14,23,30,31}$.

Outros achados e conclusões, anteriormente relatados, devem ser levados em consideração quando se encontram resultados divergentes para as medidas de fonte glótica em estudos diferentes. Essas informações incluem: a necessidade de ter dados normatizados para cada programa utilizado, a importância de verificar a vogal utilizada na emissão e a posição do microfone, o conhecimento da grande sensibilidade do método de análise acústica e que diferentes algoritmos são utilizados, conforme o software utilizado ${ }^{3,9,17,26}$. No que se refere às características espectrográficas da voz, o espectro da produção sonora de cada indivíduo é único, no entanto existem determinadas características que podem caracterizar um grupo de sujeitos. Um estudo concluiu que as características dos formantes dos homens variam pouco em relação às suas variações de medidas físicas do corpo ${ }^{5}$. Deste modo, é possível pensar que determinados achados espectrográficos costumam ser frequentes nos homens, sendo possível uma caracterização geral para esse grupo.

\section{CONCLUSÃO}

As medidas que mais diferem entre os sexos e que podem caracterizar a voz masculina são: Jita, ShdB e NHR que são maiores nos homens, enquanto f0, PHR/HNR e ATRI são menores nos homens. Salientando-se que a fo tende a aumentar nos homens com o processo de envelhecimento vocal.

As espectrografias das vozes masculinas consideradas normais tendem a apresentar formantes $e$ harmônicos graves, na região inferior do espectro, e com média de alcance menor, podendo apresentar escurecimento variável e menos regular, harmônicos menos lineares e presença de ruído. 


\section{ABSTRACT}

Background: vocal acoustic characteristics of the filter and the male measures of the vocal source. Purpose: to accomplish a literature review describing and discussing the aspects that involve the vocal acoustic characteristic in men with normal laryngeal related to measures of the source and the vocal filter. Conclusion: the measures related to Jita, ShdB and NRH tend to be bigger in men, when fO, PHR/HNR and ATRI tend to be smaller. Spectrographies tend to show deep formants with darker variation and less regular than in women.

KEYWORDS: Voice; Men's Health; Speech Acoustic

\section{REFERÊNCIAS}

1. Pontes PAL, Vieira VP, Gonçalves MIR, Pontes AAL. Características das vozes roucas, ásperas e normais: análise acústica espectrográfica comparativa. Rev Bras Otorrinolaringol. 2002; 68(2):182-8.

2. Gonzáles J, Cervera T, Miralles JL. Análisis acústico de la voz: fiabilidad de um conjunto de parámetros multidemensionales. Acta Otorrinolaringol Esp. 2002; 53:256-68.

3. Araújo AS, Grellet M, Pereira JC, Rosa MO. Normatização de medidas acústicas da voz normal. Rev Bras Otorrinolaringol. 2002; 68(4):540-4.

4. Barros APB, Carrara-De Angelis E. Análise acústica da voz. In: Dedivitis RA, Barros APB. Métodos de avaliação e diagnóstico da laringe e voz. São Paulo: Lovise; 2002. p. 200-21.

5. González J. Formant frequencies and body size of speaker: a weak relationship in adults humans. J Phonet. 2004; 32(2):277-87.

6. Xue SA, Hao GJP, Mayo R. Volumetric measurements of vocal tracts for male speakers from different races. Clin Linguist Phonet. 2006; 20(9):691-702.

7. Filho JAX, Melo ECM, Carneiro, CG, Tsuji DH, Sennes LU. Correlação entre altura e as dimensões das pregas vocais. Rev Bras Otorrinolaringol. 2003; 69(3):371-4.

8. Brasil OOC, Yamasaki R, Leão SHS. Proposta de medição da posição vertical da laringe. Rev Bras Otorrinolaringol. 2005; 71(3):313-7.

9. Fukuyama EE. Análise acústica da voz captada na faringe próximo à fonte glótica através de microfone acoplado fibrolaringoscópio. Rev Bras Otorrinolaringol. 2001; 67(6):776-86.

10. Bele IV. The speaker's formant. J Voice. 2006; 20(4):555-78.

11. Magri A, Cukier-Blaj S, Karman DF, Camargo ZA. Correlatos perceptivos e acústicos dos ajustes supraglóticos na disfonia. Rev. CEFAC. 2007; 4(9):512-8.
12. Oliveira VL, Pinho SMR. A qualidade da voz e o trato vocal nos indivíduos de face curta e face longa. In: Pinho SMR. Tópicos em voz. Rio de Janeiro: Guanabara-Koogan; 2001. p. 81-8.

13. Guimarães I, Abberton E. Fundamental frequency in speakers of Portuguese for different voice samples. J Voice. 2005; 19(4):592-606.

14. Godino-Llorente JI, Osma-Ruiz V, Sáenz-Lechón N, Cobeta-Marco I, González-Herranz R, RamírezCalvo C. Acoustic analysis of voice using WPCVox: a comparative study with Multi Dimensional Voice Program. Eur Arch Otorhinolaryngol. 2008; 265(4):465-76.

15. Godino-Llorente JI, Sáenz-Lechón N, OsmaRuiz V, Aguilera-Navarro S, Gómez-Vilda P. An integrated tool for the diagnosis of voice disorders. Medical Engineering \& Physics. 2006; 28:276-89.

16. Pinho SMR, Camargo Z. Introdução à análise acústica da voz e da fala. In: Pinho SMR. Tópicos em voz. Rio de Janeiro: Guanabara-Koogan; 2001. p. 19-44.

17. Felippe ACN, Grillo MHMM, Grechi TH. Normatização de medidas acústicas para vozes normais. Rev Bras Otorrinolaringol. 2006; 72(5):659-64.

18. Smits I, Ceuppens P, Bodt MS. A comparative study of acoustic voice measurements by means of Dr. Speech and Computadorized Speech Lab. J Voice. 2005; 19(2):187-96.

19. Santos IR. Análise acústica da voz de indivíduos na terceira idade. [dissertação] São Carlos (SP): Universidade de São Paulo; 2005. $189 f$.

20. Harnsberger JD, Shirivastav R, Brown Junior WS, Rothman $\mathrm{H}$, Hollien $\mathrm{H}$. Speaking rate and fundamental frequency as speech cues to perceived age. J Voice. 2008; 22(1):58-69.

21. Mifune E, Justino VSS, Camargo Z, Gregio F. Análise acústica da voz do idoso: caracterização da freqüência fundamental. Rev. CEFAC. 2007; 9(8):238-47.

22. Behlau MS, Pontes $P$, Tosi O. Determinação da frequência fundamental e suas variações em altura 
(jitter) e intensidade (shimmer) para falantes do português brasileiro. Acta AWHO. 1985; 4(1):5-9.

23. Nicastri M, Chiarella G, Gallo LV, Catalano $\mathrm{M}$, Cassandro E. Multidimensional voice program (MDVP) and amplitude variation parameters in euphonic adult subjects. Normative study. Acta Otorhinolaryngol Ital. 2004; 24:337-41.

24. Figueiredo DC, Souza PRF, Gonçalves MIR, Biase NG. Análise perceptivo-auditiva, acústica computadorizada e laringológica da voz de adultos jovens fumantes e não-fumantes. Rev Bras Otorrinolaringol. 2003; 69(6):791-9.

25. Zhang Y, Jiang JJ. Acoustic analyses of sustained and running voices from patients with laryngeal pathologies. J Voice. 2008; 22(1):1-9.

26. Titze IR. Workshop on acoustic voice analysis. Summary Statement. lowa City: National Center for Voice and Speech; 1994.
27. Iglesia FV, González SF, Gómez MC. Evaluación espectral cualitativa de la voz esofágica. Acta Otorrinolaringol Esp. 2006; 57:319-23.

28. Lima MFB, Camargo ZA, Ferreira LP, Madureira $S$. Qualidade vocal e formantes das vogais de falantes adultos da cidade de João Pessoa. Rev. CEFAC. 2007; 9(1):99-109.

29. Kazi RA, Prasad VMN, Kanagaligam J, Nutting $\mathrm{CM}$, Clarke $\mathrm{P}$, Rhys-Evans $\mathrm{P}$, Harrington KJ. Assesment of the formant frequencies in normal and laryngectomized individuals using Linear Predictive Coding. J Voice. 2007; 21(6):661-8.

30. Wang CC, Huang HT. Voice acoustic analysis of normal Taiwanese adults. J Chin Med Assoc. 2004; 67:179-84.

31. Yaser SN, Wingate JM. Fundamental frequency characteristics of jordanian arabic speakers. J Voice. (In press). 2008.

DOI: 10.1590/S1516-18462010005000040

RECEBIDO EM: 23/08/2009

ACEITO EM: 28/01/2010

Endereço para correspondência:

Bárbara Costa Beber

Rua Juvenal Custódio Oliveira, 318

Palmares do Sul - RS

CEP: 95540-000

E-mail: bcbfono@yahoo.com.br 